\title{
Dynamics of a Stochastic Delayed Predator-Prey System with Beddington-DeAngelis Functional Response
}

\author{
Mengwei Li, Yuanfu Shao, Yafei Yang \\ College of Physics, Guilin University of Technology, Guilin, China \\ Email: 419763620@qq.com, shaoyuanfu@163.com, 1115917086@qq.com
}

How to cite this paper: Li, M.W., Shao, Y.F. and Yang, Y.F. (2019) Dynamics of a Stochastic Delayed Predator-Prey System with Beddington-DeAngelis Functional Response. International Journal of Modern Nonlinear Theory and Application, 8, 93-105. https://doi.org/10.4236/ijmnta.2019.84007

Received: September 9, 2019

Accepted: November 11, 2019

Published: November 14, 2019

Copyright $\odot 2019$ by author(s) and Scientific Research Publishing Inc. This work is licensed under the Creative Commons Attribution International License (CC BY 4.0).

http://creativecommons.org/licenses/by/4.0/

\begin{abstract}
This paper is concerned with a stochastic predator-prey system with Beddington-DeAngelis functional response and time delay. Firstly, we show that this system has a unique positive solution as this is essential in any population dynamics model. Secondly, the validity of the stochastic system is guaranteed by stochastic ultimate boundedness of the analyzed solution. Finally, by constructing suitable Lyapunov functions, the asymptotic moment estimation of the solution was given. These properties of the solution can provide theoretical support for biological resource management.
\end{abstract}

\section{Keywords}

Beddington-DeAngelis Response, Stochastic Perturbation, Stochastic Ultimate Boundedness, Asymptotic Moment Estimation

\section{Introduction}

The dynamical relationship between prey and predator has long been and will continue to be a dominant theme in ecology due to its universal importance and existence. One important component of the predator-prey is functional response, i.e. the rate of prey consumption by an average predator. The functional response can be classified into two types: predator-dependent and prey-dependent. The classical Holling types I-III [1] [2] are strictly prey-dependent functional response; The main predator-dependent functional response has Crowley-Martin type [3], Hassell-Varley type [4], as well as Beddington-DeAngelis type by Beddington [5] and DeAngelis et al. [6]. There is much significant evidence to suggest that Beddington-DeAngelis functional response occurs quite frequently in natural systems and laboratory (see e.g. [7] [8]). The classical predator-prey model with 
Beddington-DeAngelis functional response can be expressed as follows

$$
\left\{\begin{array}{l}
\mathrm{d} x(t)=x(t)\left[a_{1}-b_{1} x\left(t_{1}\right)-\frac{c_{1} y}{1+m_{1} x(t)+m_{2} y(t)}\right] \mathrm{d} t \\
\mathrm{~d} y(t)=y(t)\left[a_{2}-b_{2} y(t)+\frac{c_{2} x(t)}{1+m_{1} x(t)+m_{2} y(t)}\right] \mathrm{d} t .
\end{array}\right.
$$

where $x(t)$ and $y(t)$ represent the size of the prey and predator populations at time $t$, respectively. The parameter $a_{1}$ denotes the intrinsic growth rate of the prey population and $a_{2}$ denotes the death date of the predator population. The parameter $b_{1}$ and $b_{2}$ are the density-dependent coefficients of the prey and predator populations, respectively. The parameter $c_{1}$ and $c_{2}$ represent the capturing rate of the predator and the rate of conversion of nutrients into the reproduction for the predator, respectively.

However, the model is deterministic, and does not incorporate the effect of environmental noise, which is always present. In the real world, population models are always affected by the environmental noise, which is an important component in an ecosystem [9] [10]. Thus, it is interesting to study how the environmental noise affects the population models. To fit the reality better, many authors have introduced white noise into the population dynamics to reveal the effects of the white noise [11] [12]. Inspired by the above facts, in this paper, we assume that fluctuations in the environment mainly affect the intrinsic growth rate $a_{1}$ and the death rate $a_{2}$, that is

$$
a_{1} \rightarrow a_{1}+\alpha_{1} \dot{W}_{1}(t), a_{2} \rightarrow a_{2}+\alpha_{2} \dot{W}_{2}(t) .
$$

Then we obtain the following stochastic system

$$
\left\{\begin{array}{l}
\mathrm{d} x(t)=x(t)\left[a_{1}-b_{1} x(t)-\frac{c_{1} y}{1+m_{1} x(t)+m_{2} y(t)}\right] \mathrm{d} t+\alpha_{1} x(t) \mathrm{d} W_{1}(t), \\
\mathrm{d} y(t)=y(t)\left[a_{2}-b_{2} y(t)+\frac{c_{2} x(t)}{1+m_{1} x(t)+m_{2} y(t)}\right] \mathrm{d} t+\alpha_{2} y(t) \mathrm{d} W_{2}(t) .
\end{array}\right.
$$

On the other hand, more realistic and interesting models of population interactions should take the effects of time delay into account [13] [14] [15] [16]. In general, delay differential equations can exhibit much more complicated dynamics than differential equations without delay. Liu [17] has investigated global asymptotic stability of the positive equilibrium about stochastic predator-prey system with Beddingtons-DeAngelis and time delay. However, so far as we know a very little amount of work has been done with the stochastic predator-prey system with Beddingtons-DeAngelis and time delay. Therefore it is interesting and important to study the following stochastic delayed predator-prey model with Beddington-DeAngelis functional response.

$$
\left\{\begin{array}{l}
\mathrm{d} x(t)=x(t)\left[a_{1}-b_{1} x\left(t-\tau_{1}\right)-\frac{c_{1} y}{1+m_{1} x(t)+m_{2} y(t)}\right] \mathrm{d} t+\alpha_{1}(t) \mathrm{d} W_{1}(t), \\
\mathrm{d} y(t)=y(t)\left[a_{2}-b_{2} y\left(t-\tau_{2}\right)+\frac{c_{2} x\left(t-\tau_{3}\right)}{1+m_{1} x\left(t-\tau_{3}\right)+m_{2} y\left(t-\tau_{3}\right)}\right] \mathrm{d} t+\alpha_{2} y(t) \mathrm{d} W_{2}(t) .
\end{array}\right.
$$


with the initial conditions

$$
x_{0}(\theta)=\phi_{1}(\theta)>0, \quad y_{0}(\theta)=\phi_{2}(\theta)>0, \theta \in[-\tau, 0], \tau=\max \left\{\tau_{1}, \tau_{2}, \tau_{3}\right\} .
$$

where $\tau>0$ denotes the delay;

$$
\begin{gathered}
\phi=\left(\phi_{1}, \phi_{2}\right) \in C\left([-\tau, 0], R_{+}^{2}\right), R_{+}^{2}=\{(x, y): x \geq 0, y \geq 0\}, \\
\|\phi\|=\max \{|\phi(\theta)|: \theta \in[-\tau, 0]\} .
\end{gathered}
$$

and $|\phi|$ is any norm in $R_{+}^{2}$. As usual, we use the notation $x_{t}(\theta)=x(t+\theta)$ for $\theta \in[-\tau, 0]$.

The rest of the paper is organized as follows. In Section 2, we show that system (1.3) has a global positive solution. In Section 3, stochastic ultimate boundedness is studied. In Section 4, we investigate the asymptotic moment estimation. In Section 5, we present numerical simulations to illustrate our mathematical findings. We close the paper with conclusions and discussions in Section 6.

\section{Global Positive Solutions}

Throughout this paper, unless otherwise specified, let $\left(\Omega,\left\{\mathcal{F}_{t}\right\}_{t \geq 0}, \mathcal{P}\right)$ be a complete probability space with a filtration $\left\{\mathcal{F}_{t}\right\}_{t \geq 0}$ satisfying the usual conditions (i.e. it is right continuous and $\mathcal{F}_{0}$ contains all $\mathcal{P}$-null sets). Moreover, let $W_{i}(t),(i=1,2)$ be standard Brownian motions defined on this probability space. Also let $R_{+}^{n}=\left\{x \in R^{n}: x_{i}>0\right.$ for all $\left.1 \leq i \leq n\right\}$.

In order for a stochastic differential equation to have a global solution for any given initial condition, it is generally necessary to data the coefficients of the equation are generally required to satisfy the liner growth condition and local Lipschitz condition (see e.g. [18]). However, the coefficients of (1.3) neither obey the linear growth condition nor local Lipschitz condition. The existence of local positive solutions is given by variable substitution and Itồs formula.

Lemma 2.1. For any initial value $\{(x(t), y(t)):-\tau \leq t \leq 0\} \in C\left([-\tau, 0] ; R_{+}^{2}\right)$, there is a unique positive local solution $(x(t), y(t)), t \in\left[-\tau, \tau_{e}\right)$ of system (1.3), where $\tau=\max \left\{\tau_{1}, \tau_{2}, \tau_{3}\right\}$ and $\tau_{e}$ is the explosion time.

Proof. Consider the following system

$$
\left\{\begin{array}{l}
\mathrm{d} f(t)=\left[a_{1}-b_{1} \mathrm{e}^{f\left(t-\tau_{1}\right)}-\frac{1}{2} \alpha_{1}^{2} \mathrm{e}^{2 f(t)}-\frac{c_{1} \mathrm{e}^{g(t)}}{1+m_{1} \mathrm{e}^{f(t)}+m_{2} \mathrm{e}^{g(t)}}\right] \mathrm{d} t+\alpha_{1} \mathrm{~d} W_{1}(t), \\
\mathrm{d} g(t)=\left[a_{2}-b_{2} \mathrm{e}^{g\left(t-\tau_{2}\right)}-\frac{1}{2} \alpha_{2}^{2} \mathrm{e}^{2 g(t)}+\frac{c_{2} \mathrm{e}^{f\left(t-\tau_{3}\right)}}{1+m_{1} \mathrm{e}^{f\left(t-\tau_{3}\right)}+m_{2} \mathrm{e}^{g\left(t-\tau_{3}\right)}}\right] \mathrm{d} t+\alpha_{2} \mathrm{~d} W_{2}(t) .
\end{array}\right.
$$

with initial value $f(0)=\log x_{0}, g(0)=\log y_{0}$. It is clear that the coefficient of system (2.1) satisfy local Lipschitz condition, then there is an unique local solution $(f(t), g(t)), t \in\left[0, \tau_{e}\right)$ of system (2.1). Therefore, by Itô’s formula, it is easy to find that $\left(x(t)=\mathrm{e}^{f(t)}, y(t)=\mathrm{e}^{g(t)}\right)$ is the unique positive local solution of the system (1.3) with the initial value $x_{0}>0, y_{0}>0$.

Next, give the existence of the positive solution.

Theorem 2.1. For any given initial value 


$$
\{(x(t), y(t)):-\tau \leq t \leq 0\} \in C\left([-\tau, 0] ; R_{+}^{2}\right),
$$

there is a unique solution $(x(t), y(t))$ of system (1.3) on $t \geq 0$, and the solution will remain in $R_{+}^{2}$ with probability 1 .

Proof. Since, Lemma 2.1 shows that there is a positive local solution $(x(t), y(t)), t \in\left[0, \tau_{e}\right)$ of system (1.3), then to show this solution is global, we only need to show that $\tau_{e}=\infty$,a.s., Let $m_{0} \geq 0$ be sufficiently large so that both $x_{0}$ and $y_{0}$ lie within the interval $\left[1 / u_{0}, u_{0}\right]$. For each integer $u>u_{0}$, define the stopping time

$$
\tau_{u}=\inf \left\{t \in\left[0, \tau_{e}\right): x(t) \notin(1 / u, u) \text {, or } y(t) \notin(1 / u, u)\right\} .
$$

where throughout this paper, we set $\inf \Phi=\infty$ (as usual $\Phi$ denotes the empty set). Clearly, $\tau_{u}$ is increasing as $u \rightarrow \infty$. Set $\tau_{\infty}=\lim _{u \rightarrow \infty} \tau_{u}$, Whence $\tau_{\infty}<\tau_{e}$,a.s. If we can show that $\tau_{\infty}=\infty$, a.s., Then $\tau_{e}=\infty$ and $(x(t), y(t)) \in R_{+}^{2}$, a.s. . For if this statement is false, then there are a pair of constants $T>0$ and $\varepsilon \in(0,1)$, such that

$$
P\left\{\tau_{u} \leq T\right\}>\varepsilon .
$$

Hence there is an integer $U_{1} \geq U_{0}$ such that

$$
P\left\{\tau_{u} \leq T\right\} \geq \varepsilon, u \geq U_{1} .
$$

Define a $C^{2}$-function $V: R_{+}^{2} \rightarrow \bar{R}_{+}$by

$$
\begin{gathered}
V_{1}(x(t), y(t))=(\sqrt{x}-1-0.5 \log x)+(\sqrt{y}-1-0.5 \log y) . \\
V_{2}(x(t), y(t))=V_{1}(x(t), y(t))+\int_{t-\tau_{1}}^{t}|x(s)|^{2} \mathrm{~d} s+\int_{t-\tau_{2}}^{t}|y(s)|^{2} \mathrm{~d} s .
\end{gathered}
$$

The non-negativity of $V_{1}(x(t), y(t))$ can be seen from

$$
\sqrt{u}-1-0.5 \log u \geq 0, \forall u>0 \text {. }
$$

Using Itồs formula, we get

$$
\begin{aligned}
& \mathrm{d} V_{2}=\mathrm{d}\left[V_{1}(x(t), y(t))+\int_{t-\tau_{1}}^{t}|x(s)|^{2} \mathrm{~d} s+\int_{t-\tau_{2}}^{t}|y(s)|^{2} \mathrm{~d} s\right] \\
& =0.5\left(x^{-0.5}(t)-x^{-1}(t)\right) x(t)\left[\left(a_{1}-b_{1} x\left(t-\tau_{1}\right)\right.\right. \\
& \left.\left.-\frac{c_{1} y(t)}{1+m_{1} x(t)+m_{2} y(t)}\right) \mathrm{d} t+\alpha_{1} \mathrm{~d} W_{1}(t)\right] \\
& +0.5 \alpha_{1}^{2} x^{2}(t)\left(-0.25 x^{-1.5}(t)+0.5 x^{-2}(t)\right) \mathrm{d} t \\
& +0.5\left(y^{-0.5}(t)-y^{-1}(t)\right) y(t)\left[\left(a_{2}-b_{2} y\left(t-\tau_{1}\right)\right.\right. \\
& \left.\left.-\frac{c_{2} x\left(t-\tau_{3}\right)}{1+m_{1} x\left(t-\tau_{3}\right)+m_{2} y\left(t-\tau_{3}\right)}\right) \mathrm{d} t+\alpha_{2} \mathrm{~d} W_{2}(t)\right] \\
& +0.5 \alpha_{2}^{2} y^{2}(t)\left(-0.25 y^{-1.5}(t)+0.5 y^{-2}(t)\right) \mathrm{d} t \\
& +\left[|x(t)|^{2}-\left|x\left(t-\tau_{1}\right)\right|^{2}+|y(t)|^{2}-\left|y\left(t-\tau_{2}\right)\right|^{2}\right] \mathrm{d} t
\end{aligned}
$$




$$
\begin{aligned}
& =0.5\left(x^{0.5}(t)-1\right)\left[\left(a_{1}-b_{1} x\left(t-\tau_{1}\right)-\frac{c_{1} y(t)}{1+m_{1} x(t)+m_{2} y(t)}\right) \mathrm{d} t+\alpha_{1} \mathrm{~d} W_{1}(t)\right] \\
& +0.5 \alpha_{1}^{2}\left(-0.25 x^{0.5}(t)+0.5\right) \mathrm{d} t+0.5\left(y^{0.5}(t)-1\right)\left[\left(a_{2}-b_{2} y\left(t-\tau_{1}\right)\right.\right. \\
& \left.\left.-\frac{c_{2} x\left(t-\tau_{3}\right)}{1+m_{1} x\left(t-\tau_{3}\right)+m_{2} y\left(t-\tau_{3}\right)}\right) \mathrm{d} t+\alpha_{2} \mathrm{~d} W_{2}(t)\right] \\
& +0.5 \alpha_{2}^{2}\left(-0.25 y^{0.5}(t)+0.5\right) \mathrm{d} t \\
& +\left[|x(t)|^{2}-\left|x\left(t-\tau_{1}\right)\right|^{2}+|y(t)|^{2}-\left|y\left(t-\tau_{2}\right)\right|^{2}\right] \mathrm{d} t \\
& =\left\{|x(t)|^{2}-\left|x\left(t-\tau_{1}\right)\right|^{2}+0.5\left[a_{1} x^{0.5}(t)-b_{1} x\left(t-\tau_{1}\right) x^{0.5}(t)\right.\right. \\
& \left.-\frac{c_{1} y(t) x^{0.5}(t)}{1+m_{1} x(t)+m_{2} y(t)}-a_{1}+\frac{c_{1} y(t)}{1+m_{1} x(t)+m_{2} y(t)}-0.25 \alpha_{1}^{2} x^{0.5}(t)+0.5 \alpha_{1}^{2}\right] \\
& \left.+0.5 b_{1} x\left(t-\tau_{1}\right)\right\} \mathrm{d} t+\left\{|y(t)|^{2}-\left|y\left(t-\tau_{2}\right)\right|^{2}\right. \\
& +0.5\left[a_{2} y^{0.5}(t)-b_{2} y\left(t-\tau_{2}\right) y^{0.5}(t)+\frac{c_{2} x\left(t-\tau_{3}\right) y^{0.5}(t)}{1+m_{1} x\left(t-\tau_{3}\right)+m_{2} y\left(t-\tau_{3}\right)}-a_{2}\right. \\
& \left.\left.-\frac{c_{2} x\left(t-\tau_{3}\right) y^{0.5}(t)}{1+m_{1} x\left(t-\tau_{3}\right)+m_{2} y\left(t-\tau_{3}\right)}-0.25 \alpha_{2}^{2} y^{0.5}(t)+0.5 \alpha_{2}^{2}\right]+0.5 b_{2} y\left(t-\tau_{2}\right)\right\} \mathrm{d} t \\
& +0.5\left(x^{0.5}(t)-1\right) \mathrm{d} W_{1}(t)+0.5\left(y^{0.5}(t)-1\right) \mathrm{d} W_{2}(t) \\
& \leq\left\{|x(t)|^{2}-\left|x\left(t-\tau_{1}\right)\right|^{2}+0.5\left[a_{1} x^{0.5}(t)+\frac{c_{1} y(t)}{1+m_{1} x(t)+m_{2} y(t)}\right.\right. \\
& \left.\left.-0.25 \alpha_{1}^{2} x^{0.5}(t)+0.5 \alpha_{1}^{2}\right]+\left(0.25 b_{1}\right)^{2}+\left|x\left(t-\tau_{1}\right)\right|^{2}\right\} \mathrm{d} t \\
& +\left\{|y(t)|^{2}-\left|y\left(t-\tau_{2}\right)\right|^{2}+0.5\left[a_{2} y^{0.5}(t)+\frac{c_{2} x\left(t-\tau_{3}\right) y^{0.5}(t)}{1+m_{1} x\left(t-\tau_{3}\right)+m_{2} y\left(t-\tau_{3}\right)}\right.\right. \\
& \left.\left.-0.25 \alpha_{2}^{2} y^{0.5}(t)+0.5 \alpha_{2}^{2}\right]+\left(0.25 b_{2}\right)^{2}+\left|y\left(t-\tau_{2}\right)\right|^{2}\right\} \mathrm{d} t \\
& +0.5\left(x^{0.5}(t)-1\right) \alpha_{1} \mathrm{~d} W_{1}(t)+0.5\left(y^{0.5}(t)-1\right) \alpha_{2} \mathrm{~d} W_{2}(t) \\
& \leq\left\{|x(t)|^{2}+0.5\left[a_{1} x^{0.5}(t)+c_{1} / m_{2}+0.5 \alpha_{1}^{2}+0.125 b_{1}^{2}-0.25 \alpha_{1}^{2} x^{0.5}(t)\right]\right\} \mathrm{d} t \\
& +\left\{|y(t)|^{2}+0.5\left[a_{2} y^{0.5}(t)+c_{2} y^{0.5}(t) / m_{1}+0.5 \alpha_{2}^{2}+0.125 b_{2}^{2}-0.25 \alpha_{2}^{2} y^{0.5}(t)\right]\right\} \mathrm{d} t \\
& +0.5\left(x^{0.5}(t)-1\right) \alpha_{1} \mathrm{~d} W_{1}(t)+0.5\left(y^{0.5}(t)-1\right) \alpha_{2} \mathrm{~d} W_{2}(t) \\
& =M(x(t), y(t)) \mathrm{d} t+0.5\left(x^{0.5}(t)-1\right) \alpha_{1} \mathrm{~d} W_{1}(t)+0.5\left(y^{0.5}(t)-1\right) \alpha_{2} \mathrm{~d} W_{2}(t) \text {. }
\end{aligned}
$$

$$
\begin{aligned}
& M(x(t), y(t)) \\
& =\left\{|x(t)|^{2}+0.5\left[a_{1} x^{0.5}(t)+c_{1} / m_{2}+0.5 \alpha_{1}^{2}+0.125 b_{1}^{2}-0.25 \alpha_{1}^{2} x^{0.5}(t)\right]\right\} \\
& +\left\{|y(t)|^{2}+0.5\left[a_{2} y^{0.5}(t)+c_{2} y^{0.5}(t) / m_{1}+0.5 \alpha_{2}^{2}+0.125 b_{2}^{2}-0.25 \alpha_{2}^{2} y^{0.5}(t)\right]\right\} .
\end{aligned}
$$


which implies that

$$
M(x(t), y(t)) \leq M^{*},
$$

Because next inequality exists, we can get (2.3)

$$
\begin{aligned}
& \frac{1}{2} b_{1} x\left(t-\tau_{1}\right) \leq\left(\frac{1}{4} b_{1}\right)^{2}+\left|x\left(t-\tau_{1}\right)\right|^{2}, \\
& \frac{1}{2} b_{2} y\left(t-\tau_{2}\right) \leq\left(\frac{1}{4} b_{2}\right)^{2}+\left|y\left(t-\tau_{2}\right)\right|^{2} .
\end{aligned}
$$

To sum up, we can get

$$
\begin{aligned}
& \mathrm{d} V_{2}(x(t), y(t)) \\
& =\mathrm{d}\left[V_{1}(x(t), y(t))+\int_{t-\tau_{1}}^{t}|x(s)|^{2} \mathrm{~d} s+\int_{t-\tau_{2}}^{t}|y(s)|^{2} \mathrm{~d} s\right] \\
& \leq M(x(t), y(t))+0.5\left(x^{0.5}(t)-1\right) \alpha_{1} \mathrm{~d} W_{1}(t)+0.5\left(y^{0.5}(t)-1\right) \alpha_{2} \mathrm{~d} W_{2}(t) .
\end{aligned}
$$

Integrating both sides of the above inequality from 0 to $\tau_{m} \wedge T$ and then taking the expectations leads to

$$
\mathrm{E}\left\{\int_{0}^{\tau_{u} \wedge T} \mathrm{~d}\left[V_{1}(x(t), y(t))+\int_{t-\tau_{1}}^{t}|x(s)|^{2} \mathrm{~d} s+\int_{t-\tau_{2}}^{t}|y(s)|^{2} \mathrm{~d} s\right]\right\} \leq M^{*} \mathrm{E}\left(\tau_{u} \wedge T\right),
$$

So

$$
\begin{aligned}
& \mathrm{E}\left[\int_{\tau_{u} \wedge T-\tau_{1}}^{\tau_{u} \wedge T}|x(s)|^{2} \mathrm{~d} s+\int_{\tau_{u} \wedge T-\tau_{2}}^{\tau_{u} \wedge T}|y(s)|^{2} \mathrm{~d} s\right]+\mathrm{E}\left[x\left(\tau_{u} \wedge T\right), y\left(\tau_{u} \wedge T\right)\right] \\
& \leq \mathrm{E} \int_{-\tau_{1}}^{0}|x(s)|^{2} \mathrm{~d} s+\mathrm{E} \int_{-\tau_{2}}^{0}|y(s)|^{2} \mathrm{~d} s+V_{1}(x(0), y(0))+M^{*} \mathrm{E}\left(\tau_{u} \wedge T\right),
\end{aligned}
$$

Hence

$$
\begin{aligned}
& \mathrm{E}\left[x\left(\tau_{u} \wedge T\right), y\left(\tau_{u} \wedge T\right)\right] \\
& \leq \mathrm{E} \int_{-\tau_{1}}^{0}|x(s)|^{2} \mathrm{~d} s+\mathrm{E} \int_{-\tau_{2}}^{0}|y(s)|^{2} \mathrm{~d} s+V_{1}(x(0), y(0))+M^{*} T<+\infty
\end{aligned}
$$

Set $\Omega_{u}=\left\{\tau_{u} \leq T\right\}$ for $u \geq U_{1}$, then by (2.2), we know $P\left(\Omega_{u}\right) \geq \varepsilon$. Note that for every $\omega \in \Omega_{u}$, there is at last one of $x\left(\tau_{u}, \omega\right), y\left(\tau_{u}, \omega\right)$ equal either $u$ or $1 / u$, then $V_{1}\left(x\left(\tau_{u}\right), y\left(\tau_{u}\right)\right)$ is no less then $\min \left\{(u-1-\log u),\left(\frac{1}{u}-1+\log u\right)\right\}$.

It then follows from (2.2) and (2.5) that

$$
\begin{aligned}
& \mathrm{E} \int_{-\tau_{1}}^{0}|x(s)|^{2} \mathrm{~d} s+\mathrm{E} \int_{-\tau_{2}}^{0}|y(s)|^{2} \mathrm{~d} s+V_{1}(x(0), y(0))+M^{*} \mathrm{E}\left(\tau_{u} \wedge T\right) \\
& \geq E\left[1_{\Omega_{u}(\omega)}\left(x\left(\tau_{u}, \omega\right), y\left(\tau_{u}, \omega\right)\right)\right] \geq \varepsilon \min \left\{(u-1-\log u),\left(\frac{1}{u}-1+\log u\right)\right\} .
\end{aligned}
$$

where $1_{\Omega_{u}(\omega)}$ is the indicator function of $\Omega_{u}$. Letting $u \rightarrow \infty$ leads to the contradiction that

$$
+\infty>\mathrm{E} \int_{-\tau_{1}}^{0}|x(s)|^{2} \mathrm{~d} s+\mathrm{E} \int_{-\tau_{2}}^{0}|y(s)|^{2} \mathrm{~d} s+V_{1}(x(0), y(0))+M^{*} \mathrm{E}\left(\tau_{u} \wedge T\right)=+\infty .
$$

So we must have $\tau_{\infty}=\infty$, a.s. .

\section{Stochastic Ultimate Boundedness}

Define 3.1. The solution of system (1.3) is random and ultimately bounded, if 
there exists an any positive constant $H=H(\varepsilon)$ so that for any initial value $\{(x(t), y(t)):-\tau \leq t \leq 0\} \in C\left([-\tau, 0] ; R_{+}^{2}\right)$, it satisfies

$$
\limsup _{t \rightarrow \infty} P\{|x(t), y(t)|>H\}<\varepsilon \text {. }
$$

Lemma 3.1. For any initial value $\{(x(t), y(t)):-\tau \leq t \leq 0\} \in C\left([-\tau, 0] ; R_{+}^{2}\right)$, $(x(t), y(t))$ is a solution of the system (1.3), there exists positive constants $H(\rho), 0<\rho<1$ satisfies

$$
\limsup _{t \rightarrow \infty} E|(x(t), y(t))|^{\rho}=H(\rho) .
$$

Proof. Define $V_{3}(x)=x^{\rho}(t)+y^{\rho}(t)$, If $(x(t), y(t)) \in R_{+}^{2}$, we have

$$
\mathrm{d} V_{3}(x(t), y(t))=L V(x(t), y(t)) \mathrm{d} t+\rho \alpha_{1} x^{\rho}(t) \mathrm{d} W_{1}(t)+\rho \alpha_{2} y^{\rho}(t) \mathrm{d} W_{2}(t) .
$$

where

$$
\begin{aligned}
& L V_{3}(x(t), y(t)) \\
& =\rho x^{\rho}\left[a_{1}-b_{1} x\left(t-\tau_{1}\right)-\frac{c_{1} y(t)}{1+m_{1} x(t)+m_{2} y(t)}\right]+\frac{\rho(\rho-1)}{2} \alpha_{1}^{2} x^{\rho}(t) \\
& +\rho y^{\rho}\left[a_{2}-b_{2} y\left(t-\tau_{2}\right)+\frac{c_{2} y\left(t-\tau_{3}\right)}{1+m_{1} x\left(t-\tau_{3}\right)+m_{2} y\left(t-\tau_{3}\right)}\right]+\frac{\rho(\rho-1)}{2} \alpha_{2}^{2} y^{\rho}(t) \\
& \leq a_{1} \rho x^{\rho}(t)-\frac{\rho(1-\rho) \alpha_{1}^{2}}{2} x^{\rho}(t)+a_{2} \rho y^{\rho}(t) \\
& +\frac{c_{2} \rho x\left(t-\tau_{3}\right) y^{\rho}(t)}{1+m_{1} x\left(t-\tau_{3}\right)+m_{2} y\left(t-\tau_{3}\right)}-\frac{\rho(1-\rho) \alpha_{2}^{2}}{2} y^{\rho}(t) .
\end{aligned}
$$

Because of $0<\rho<1, \quad \rho(1-\rho)>0$, so

$$
\begin{aligned}
& L V_{3}(x(t), y(t)) \\
& \leq a_{1} \rho x^{\rho}(t)-\frac{\rho(1-\rho) \alpha_{1}^{2}}{2} x^{\rho}(t)+x^{\rho}(t)+a_{2} \rho y^{\rho}(t)-\frac{\rho(1-\rho) \alpha_{2}^{2}}{2} y^{\rho}(t) \\
& \quad+\frac{c_{2} \rho x\left(t-\tau_{3}\right) y^{\rho}(t)}{1+m_{1} x\left(t-\tau_{3}\right)+m_{2} y\left(t-\tau_{3}\right)}+y^{\rho}(t)-V_{3}(x(t), y(t)) \\
& \leq H-V_{3}(x(t), y(t)) .
\end{aligned}
$$

where $H$ is a positive number, substitute it into Equation (3.2) to get

$$
\begin{aligned}
& \mathrm{d} V_{3}(x(t), y(t)) \\
& \leq\left[H-V_{3}(x(t), y(t))\right] \mathrm{d} t+\rho \alpha_{1} x^{\rho}(t) d W_{1}(t)+\rho \alpha_{2} y^{\rho}(t) d W_{2}(t) .
\end{aligned}
$$

Applying Itô’s formula again, get

$$
\begin{aligned}
& \mathrm{d}\left[\mathrm{e}^{t} V_{3}(x(t), y(t))\right] \\
& =\mathrm{e}^{t}\left[V_{3}(x(t), y(t))+\mathrm{d} V_{3}(x(t), y(t))\right] \\
& \leq \mathrm{e}^{t} H \mathrm{~d} t+\rho \alpha_{1} \mathrm{e}^{t} x^{\rho}(t) \mathrm{d} W_{1}(t)+\rho \alpha_{2} \mathrm{e}^{t} y^{\rho}(t) \mathrm{d} W_{2}(t) .
\end{aligned}
$$

Taking the expectation of both sides of above inequality (3.2)

$$
\mathrm{e}^{t} \mathrm{E} V_{3}(x(t), y(t)) \leq V_{3}(x(0), y(0))+H\left(\mathrm{e}^{t}-1\right) .
$$


Namely

$$
\limsup _{t \rightarrow \infty} \mathrm{E} V_{3}(x(t), y(t)) \leq H
$$

And because

$$
|(x(t), y(t))|^{\theta} \leq 2^{\rho / 2} \max \left\{x^{\rho}(t), y^{\rho}(t)\right\} \leq 2^{\rho / 2} V_{3}(x(t), y(t)),
$$

So

$$
\limsup _{t \rightarrow \infty} \mathrm{E}|(x(t), y(t))|^{\rho} \leq 2^{\rho / 2} \limsup _{t \rightarrow \infty} \mathrm{E} V_{3}(x(t), y(t)) \leq 2^{\rho / 2} H \text {. }
$$

Therefore

$$
\limsup _{t \rightarrow \infty} \mathrm{E}|(x(t), y(t))|^{\rho} \leq H_{1} \text {, }
$$

Among them

$$
H_{1}=2^{\rho / 2} H \text {. }
$$

Further considering the stochastic ultimate boundedness of the solution, the following propreties hold true.

Theorem 3.1. The solution of system (1.3) is finally bounded by randomness.

Proof. Applying Lemma 3.1, set $\rho=1 / 2$, then there exists $K>0$, so that

$$
\limsup _{t \rightarrow \infty} \mathrm{E}|(x(t), y(t))|^{\frac{1}{2}} \leq K \text {. }
$$

For any $\varepsilon>0$, setting $H_{1}=[K / \varepsilon]^{\frac{1}{\rho}}$, an application of Chebyshev's inequality, there is

$$
P\left\{|(x(t), y(t))|>H_{1}\right\}<\frac{E\left[|(x(t), y(t))|^{\rho}\right]}{H_{1}^{\rho}} \leq \varepsilon,
$$

So

$$
\limsup _{t \rightarrow \infty} P\left\{|(x(t), y(t))|>H_{1}\right\} \leq K \cdot \frac{\varepsilon}{K}=\varepsilon \text {. }
$$

Namely

$$
\limsup _{t \rightarrow \infty} P\left\{|(x(t), y(t))| \leq H_{1}\right\}>1-\varepsilon \text {. }
$$

which is the desired assertion.

\section{Asymptotic Moment Estimation}

Theorem 2.1 and Theorem 3.1 show that, for any given initial condition, system (1.3) has a unique global positive solution and the solution is random and finally has upper bounded. The asymptotic moment of the solution is estimated below.

Theorem 4.1. For any given $\theta \in(0,1)$, there is positive constant $K=K(\theta)$ such that the solutions of system (1.3) with the initial condition $\{(x(t), y(t)):-\tau \leq t \leq 0\} \in C\left([-\tau, 0] ; R_{+}^{2}\right)$, have the following property 


$$
\limsup _{t \rightarrow \infty} \frac{1}{T} \int_{0}^{T} E\left[x^{\theta}(s)+y^{\theta}(s)\right] \mathrm{d} s \leq K .
$$

where $K(\theta)$ is in dependent of the initial value.

Proof. Define a $C^{2}$-function

$$
V_{4}(x(t), y(t))=x^{\theta}(t)+y^{\theta}(t),(x(t), y(t)) \in R_{+}^{2} .
$$

By Itôs formula, one can see that

$$
\mathrm{d} V_{4}(x(t), y(t))=L V_{4}(x(t), y(t)) \mathrm{d} t+\theta \alpha_{1} x^{\theta}(t) \mathrm{d} W_{1}(t)+\theta \alpha_{2} y^{\theta}(t) \mathrm{d} W_{2}(t) .
$$

where

$$
\begin{aligned}
& L V_{4}(x(t), y(t)) \\
& =\theta x^{\theta}(t)\left[a_{1}-b_{1}\left(t-\tau_{1}\right)-\frac{c_{1} y(t)}{1+m_{1} x(t)+m_{2} y(t)}\right]+\frac{\theta(\theta-1) \alpha_{1}^{2}}{2} x^{\theta}(t) \\
& +\theta y^{\theta}(t)\left[a_{2}-b_{2}\left(t-\tau_{2}\right)+\frac{c_{2} x\left(t-\tau_{3}\right)}{1+m_{1} x\left(t-\tau_{3}\right)+m_{2} y\left(t-\tau_{3}\right)}\right]+\frac{\theta(\theta-1) \alpha_{2}^{2}}{2} y^{\theta}(t) \\
& \leq a_{1} \theta x^{\theta}(t)-\frac{\theta(1-\theta) \alpha_{1}^{2}}{2} x^{\theta}(t)+a_{2} \theta y^{\theta}(t) \\
& +\frac{c_{2} \theta x\left(t-\tau_{3}\right) y^{\theta}(t)}{1+m_{1} x\left(t-\tau_{3}\right)+m_{2} y\left(t-\tau_{3}\right)}-\frac{\theta(1-\theta) \alpha_{2}^{2}}{2} y^{\theta}(t) .
\end{aligned}
$$

Therefore

$$
\begin{aligned}
& L V_{4}(x(t), y(t))+\frac{\theta(1-\theta) \alpha_{1}^{2}}{4} x^{\theta}(t)+\frac{\theta(1-\theta) \alpha_{2}^{2}}{4} y^{\theta}(t) \\
& \leq a_{1} \theta x^{\theta}(t)-\frac{\theta(1-\theta) \alpha_{1}^{2}}{4} x^{\theta}(t)+a_{2} \theta y^{\theta}(t) \\
& \quad+\frac{c_{2} \theta x\left(t-\tau_{3}\right) y^{\theta}(t)}{1+m_{1} x\left(t-\tau_{3}\right)+m_{2} y\left(t-\tau_{3}\right)}-\frac{\theta(1-\theta) \alpha_{2}^{2}}{4} y^{\theta}(t) \\
& \leq M .
\end{aligned}
$$

where $M$ is a positive number, if we take $\alpha^{2}=\min \left\{\alpha_{1}^{2}, \alpha_{2}^{2}\right\}$, from (4.1), we can get

$$
\begin{aligned}
& L V_{4}(x(t), y(t))+\frac{\theta(1-\theta) \alpha^{2}}{4}\left(x^{\theta}(t)+y^{\theta}(t)\right) \\
& \leq L V_{4}(x(t), y(t))+\frac{\theta(1-\theta) \alpha_{1}^{2}}{4} x^{\theta}(t)+\frac{\theta(1-\theta) \alpha_{2}^{2}}{4} y^{\theta}(t) \leq M .
\end{aligned}
$$

Substituting Equation (4.4) into (4.2)

$$
\begin{aligned}
\mathrm{d} V_{4}(x(t), y(t)) \leq & {\left[M-\frac{\theta(1-\theta) \alpha^{2}}{4}\left(x^{\theta}(t)+y^{\theta}(t)\right)\right] \mathrm{d} t } \\
& +\theta \alpha_{1} x^{\theta}(t) \mathrm{d} W_{1}(t)+\theta \alpha_{2} y^{\theta}(t) \mathrm{d} W_{2}(t),
\end{aligned}
$$

So

$$
\begin{aligned}
& \mathrm{d} V_{4}(x(t), y(t))+\left[\frac{\theta(1-\theta) \alpha^{2}}{4}\left(x^{\theta}(t)+y^{\theta}(t)\right)\right] \mathrm{d} t \\
& \leq M \mathrm{~d} t+\theta \alpha_{1} x^{\theta}(t) \mathrm{d} W_{1}(t)+\theta \alpha_{2} y^{\theta}(t) \mathrm{d} W_{2}(t) .
\end{aligned}
$$


Integrating both sides of the above inequality from 0 to $\tau_{u} \wedge T$ and then taking the expectations leads to

$$
\mathrm{E} V_{4}(x(t), y(t))+\frac{\theta(1-\theta) \alpha^{2}}{4} \int_{0}^{T} \mathrm{E}\left[x^{\theta}(s)+y^{\theta}(s)\right] \mathrm{d} s \leq V_{4}(x(0), y(0))+M T
$$

Namely

$$
\int_{0}^{T} \mathrm{E}\left[x^{\theta}(s)+y^{\theta}(s)\right] \mathrm{d} s \leq \frac{4}{\theta(1-\theta) \alpha^{2}}(V(x(0), y(0))+M T) .
$$

Dividing both sides by $T$

$$
\frac{1}{T} \int_{0}^{T} \mathrm{E}\left[x^{\theta}(s)+y^{\theta}(s)\right] \mathrm{d} s \leq \frac{4}{\theta(1-\theta) \alpha^{2}}\left(\frac{V(x(0), y(0))}{T}+M\right) .
$$

If we set

$$
K=\frac{4 M}{\theta(1-\theta) \alpha^{2}}
$$

We get Equation (4.1), which is the desired assertion.

\section{Numerical Simulations}

Utilize the Milstein method (see e.g., [19]) to verify the theoretical results.

Considering the following discretization equations:

$$
\left\{\begin{array}{l}
\mathrm{d} x_{i+1}=x_{i}+x_{i}\left[a_{1}-b_{1} x_{\left(i-s_{1}\right)}-\frac{c_{1} y_{i}}{1+m_{1} x_{i}+m_{2} y_{i}}\right] \mathrm{d} t+\alpha_{1} x_{i} \sqrt{\Delta t} \eta_{1, i}, \\
\mathrm{~d} y_{i+1}=y_{i}+y_{i}\left[a_{2}-b_{2} y_{\left(i-s_{2}\right)}+\frac{c_{2} x_{\left(i-s_{3}\right)}}{1+m_{1} x_{\left(i-s_{3}\right)}+m_{2} y_{\left(i-s_{3}\right)}}\right] \mathrm{d} t+\alpha_{2} y_{i} \sqrt{\Delta t} \eta_{2, i} .
\end{array}\right.
$$

where $\eta_{1, i}$ and $\eta_{2, i}$ are Gaussian random variables that are independent of each other and follow the standard normal distribution $N(0,1)$. Set $\Delta t=0.01$, step length is 300 , select

$$
\begin{aligned}
& a_{1}=0.8, \quad b_{1}=0.5, \quad c_{1}=0.2, m_{1}=0.2, \tau=\max \left\{\tau_{1}, \tau_{2}, \tau_{3}\right\}, \tau=1, \\
& a_{2}=0.3, b_{2}=0.2, \quad c_{2}=0.1, m_{2}=0.1 .
\end{aligned}
$$

And assume that the parameters below are the same as above.

Suppose initial data

$$
\phi(\theta)=(0.6,0.6)
$$

Select $\alpha_{1}=\alpha_{2}=0.1$, it can be seen from Theorem 3.1 that system (5.1) is stochastic ultimate boundedness (See green line in Figure 1(a) and Figure 2(a)). In order to discuss the influence of random white noise, $\alpha_{1}=\alpha_{2}=0$ is selected to obtain the deterministic system corresponding to system (5.1), which is ultimately bounded (See red line in Figure 1(a) and Figure 2(a)). The blue lines represent the probability density functions of $x$ and $y$ at time 300 (in Figure 1(b) and Figure 2(b)). 


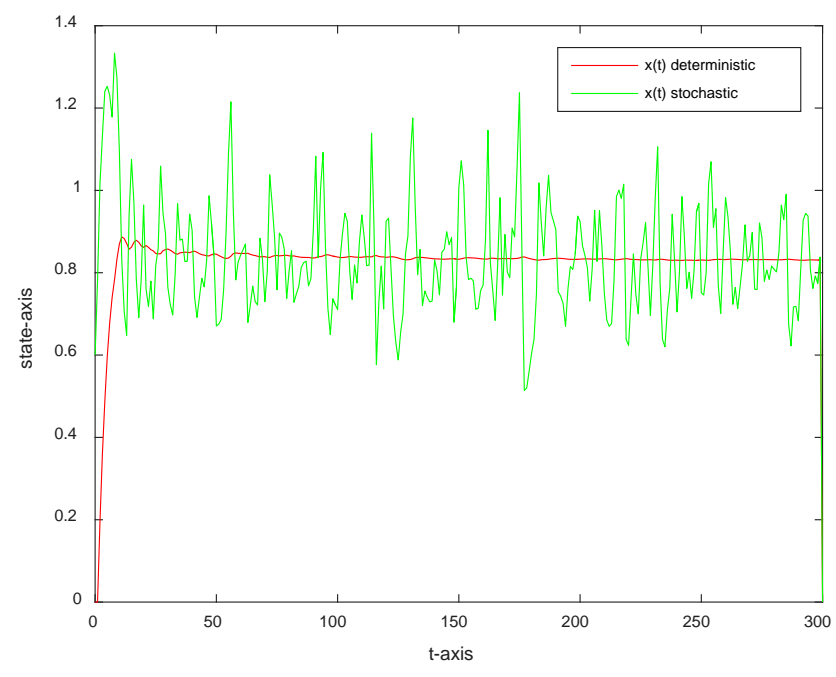

(a)

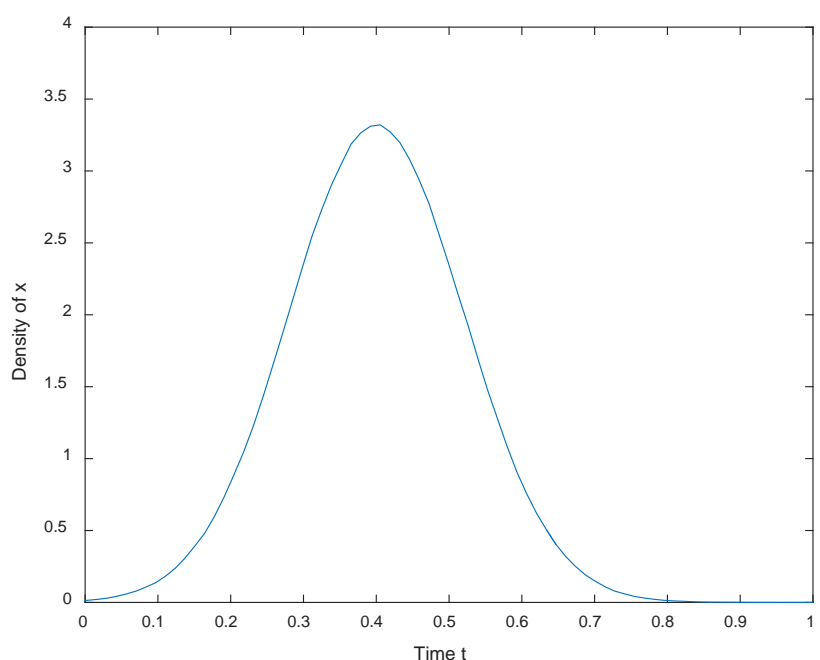

(b)

Figure 1. System (5.1) take the initial data $\phi(\theta)=(0.6,0.6)$, (a) Green line: $\alpha_{1}=\alpha_{2}=0.1$, red line: $\alpha_{1}=\alpha_{2}=0$. (b) The blue line represents the probability functions of $x$.

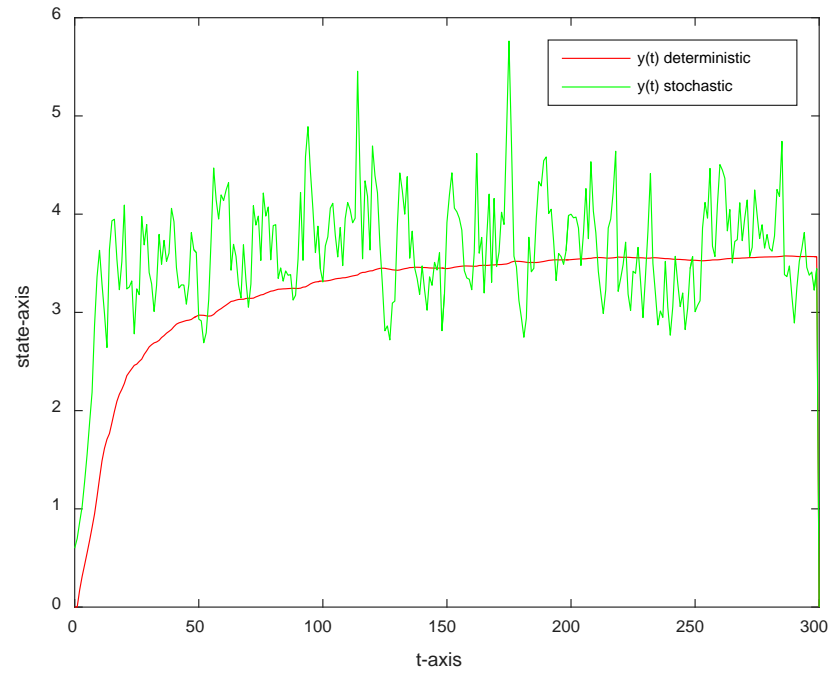

(a)

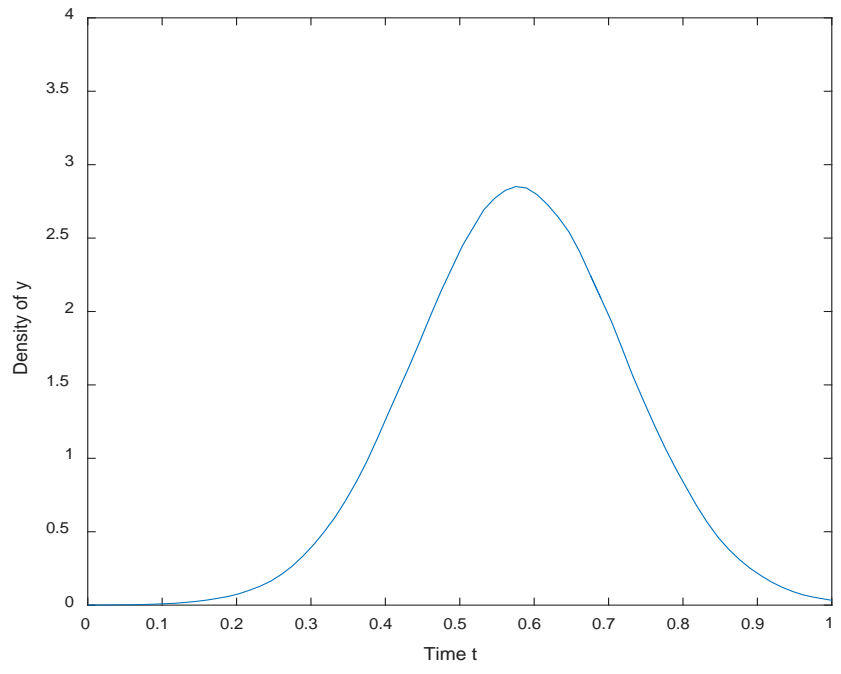

(b)

Figure 2. System (5.1) take the initial data $\phi(\theta)=(0.6,0.6)$, (a) Green line: $\alpha_{1}=\alpha_{2}=0.1$, red line: $\alpha_{1}=\alpha_{2}=0$. (b) The blue line represents the probability functions of $y$.

\section{Conclusion}

The research of predator-prey system has certainly theory and application value. In this paper, we study a stochastic delayed predator-prey system with Beddington-DeAngelis functional response and discuss some properties of the system solution, which include existence and uniqueness of the global positive solution, stochastic ultimate boundedness of the solution, and asymptotic moment estimate. These properties provide a theoretical basis for the management of population dynamic system. Based on this work, we can also study population dynamics system with time delay and other types of functional responses. 


\section{Acknowledgements}

This work was supported by the National Natural Science Foundation of China (11861027).

\section{Conflicts of Interest}

The authors declare no conflicts of interest regarding the publication of this paper.

\section{References}

[1] Holling, C.S. (1959) The Components of Predation as Revealed by a Study of Small Mammal Predation of the European Pine Sawfly. The Canadian Entomologist, 91, 293-320. https://doi.org/10.4039/Ent91293-5

[2] Holling, C.S. (1959) Some Characteristics of Simple Types of Predation and Parasitism. The Canadian Entomologist, 91, 385-395. https://doi.org/10.4039/Ent91385-7

[3] Crowley, P.H. and Martin, E.K. (1989) Functional Response and Interference within and between Year Classes of a Dragonfly Population. Journal of the North American Benthological Society, 8, 211-221. https://doi.org/10.2307/1467324

[4] Hassell, M.P. and Varley, C.C. (1969) New Inductive Population Model for Insect Parasites and Its Bearing on Biological Control. Nature, 223, 1133-1137.

https://doi.org/10.1038/2231133a0

[5] Beddington, J.R. (1975) Mutual Interference between Parasites or Predators and Its Effect on Searching Efficiency. Journal of Animal Ecology, 44, 331-341. https://doi.org/10.2307/3866

[6] DeAngelis, D.L., Goldsten, R.A. and Neill, R. (1975) A Model for Tropic Interaction. Ecology, 56, 881-892. https://doi.org/10.2307/1936298

[7] Jost, C. and Arditi, R. (2001) From Pattern to Process: Identifying Predator-Prey Interactions. Population Ecology, 43, 229-243. https://doi.org/10.1007/s10144-001-8187-3

[8] Skalski, G.T. and Gilliam, J.F. (2001) Functional Responses with Predator Interference: Viable Alternatives to the Holling Type II Model. Ecology, 82, 3083-3092. https://doi.org/10.1890/0012-9658(2001)082[3083:FRWPIV]2.0.CO;2

[9] Gard, T.C. (1984) Persistence in Stochastic Food Web Models. Bulletin of Mathematical Biology, 46, 357-370.

[10] Gard, T.C. (1988) Introduction to Stochastic Differential Equations. Dekker, New York.

[11] Mandal, P. and Bnerjee, M. (2012) Stochastic Persistence and Stationary Distribution in a Holling-Tanner Type Prey-Predator Model. Physica A, 391, 1216-1233. https://doi.org/10.1016/j.physa.2011.10.019

[12] Liu, M. and Wang, K. (2012) Persistence, Extinction and Global Asymptotical Stability of a Non-Autonomous Predator-Prey Model with Random Perturbation. Applied Mathematical Modelling, 36, 5344-5353. https://doi.org/10.1016/j.apm.2011.12.057

[13] Fan, M. and Kuang, Y. (2008) Dynamics of a Nonautonomous Predator-Prey System with the Beddington-DeAngelis Functional Response. Journal of Mathematical Analysis and Applications, 295, 15-39. https://doi.org/10.1016/j.jmaa.2004.02.038

[14] Liu, S., Beretta, E. and Breda, D. (2010) Predator-Prey Model of Beddington-DeAngelis 
Type with Maturation and Gestation Delays. Nonlinear Analysis. Real World Applications, 11, 4072-4091. https://doi.org/10.1016/j.nonrwa.2010.03.013

[15] Liu, M. and Wang, K. (2012) Global Asymptotic Stability of a Stochastic Lotka-Volterra Model with Infinite Delays, Commun. Communications in Nonlinear Science and Numerical Simulation, 17, 3115-3123. https://doi.org/10.1016/j.cnsns.2011.09.021

[16] Geritz, S. and Gyllenberg, M. (2012) A Mechanistic Derivation of the DeAngelis-Beddington Functional Response. Journal of Theoretical Biology, 314, 106-108. https://doi.org/10.1016/j.jtbi.2012.08.030

[17] Liu, M. and Bai, C. (2014) Global Asymptotic Stability of a Stochastic Delayed Predator-Prey System with Beddington-DeAngelis Functional Response. Applied Mathematics and Computation, 226, 581-588. https://doi.org/10.1016/j.amc.2013.10.052

[18] Mao, X. (1997) Stochastic Differential Equations and Applications. Horwood Publishing, Chichester.

[19] Hu, Y., Mohammed, S.E. and Yan, F. (2004) Discrete-Time Approximations of Stochastic Delay Equations: The Milstein Scheme. The Annals of Probability, 32, 265-314. https://doi.org/10.1214/aop/1078415836 